\title{
REITER'S DISEASE
}

\section{Tendency to Ultimate Spontaneous Cure}

\author{
By R. R. Willcox, M.B., B.S. \\ Physician in charge Venereal Disease Dept. King Edward Memorial Hospital, Windsor. Assistant, Venereal Disease Dept. \\ St. Mary's Hospital, London
}

That penicillin and sulphonamides have no dramatic action on Reiter's disease is generally evident and, so far, fever has given the best results. Willcox et al. ${ }^{1}$ reported a satisfactory outcome in two cases by employing gold salts though Harkness ${ }^{2}$ and others have not found them of value. Baxter ${ }^{3}$ also reported a case improving with their use but suggested that any value they may possess is of a non-specific nature. Baines ${ }^{4}$, noting the resemblance between certain aspects of Reiter's disease and so-called abacterial pyuria, considered that neoarsphenamine, though having no action on the joint manifestations, is of value for the genito-urinary lesions of the former condition.

The difficulty in assessing the results of treatment given to small numbers of patients are always great if there is any tendency to spontaneous recovery and especially in a disease possessing all grades of severity from the asymptomatic, the mild and moderate to the other end of the scale where, in the malignant forms, the greatest constitutional disturbance is met. This is exemplified in the case histories of two patients, both of whom were failures to penicillin and sulphonamides, who ultimately recovered without specific drugs or fever on a more or less ambulant basis.

\section{Case 1-Male, aged 47, a plasterer by trade}

In January, 1935, he contracted acute gonorrhoea which responded well to irrigations and a course of vaccine. His prostate was free of pus cells within 40 days and he was discharged as cured with a negative gonococcal complement fixation test after ten months. He experienced no further trouble until June 16th, 1947, when, 17 days after a risk. with a stranger he came to hospital with a slight muco-purulent urethral discharge of four-days' duration, in which no gonococci were demonstrated. On this and the two succeeding days he was given no specific treatment and repeated examinations of the discharge for gonococci and trichomonads were negative. The two glass urine tests showed haziness in the first glass only. 250,000 units of penicillin in oil-beeswax were then administered intramuscularly but there was no improvement in the urethritis when seen on the 12th day of the disease. At this time a seven-day course of sulphadiazine ( $4 \mathrm{gm}$. daily) was commenced and under its use the discharge may have lessened, but the haziness of the urine persisted. Moreover, during this time, on the 17 th day of the disease, he developed a bilateral sterile conjunctivitis which he attributed to his work as a plasterer. When seen on the 2 Ist day of the disease the conjunctivitis was still severe, the mucopurulent discharge persisted and the body temperature was $99^{\circ} \mathrm{F}$. No arthritis was observed. The ocular condition cleared within six days with argyrol drops and normal saline washes during which period he was also given three intramuscular injections of $5 \mathrm{cc}$. of his own blood. It was during this time also that he developed pain in his right hip but he did not consider that this was worth reporting until after it had cleared three weeks later. It was never severe and did not interfere with his work. By July 17 th his discharge had completely resolved and the urine was quite clear.

On July $17^{\text {th }}$ he developed penile lesions resembling a mild erosive balanitis, but two weeks. later, on the 5oth day of the disease, there were five yellow, distinctly raised, keratotic papules, each 3-5 $\mathrm{mm}$. in diameter, along the dorsum of the coronal sulcus. At this time all symptoms of the urethritis had gone, the urine was clear, no abnormalities were detectable in the prostatic smear and the blood Wassermann,. Kahn and gonococcal complement fixation tests were all negative. Examination of the joints then revealed no abnormalities. The keratosis was left untreated for nearly six weeks during which time no other symptoms developed and the serology remained persistently negative. On September I Ith, 1947, the keratosis was still unchanged and he was given an ointment containing 2 per cent. of salicylic acid, and when seen two weeks later his penis was normal.

The patient was treated throughout as an outpatient and his work was uninterrupted. 


\section{Case 2-Male, aged 41, a gardener}

In 1935 he had an urethral discharge of unknown etiology, which was followed by a synovitis of his right knee and necessitated his being absent from work for one month. On May 3rd, 1947, he consulted his private doctor on account of a urethral discharge. No sexual contact, apart from his wife, was admitted. An examination of the discharge showed no gonococci and during the week that followed he was given $20 \mathrm{gm}$. of sulphamerazine and three intramuscular injections of 250,000 units of penicillin in oil-beeswax.

On May Ioth, there being no improvement, he was referred to the clinic. At this time there was a profuse muco-purulent urethral discharge in which neither gonococci or trichomonads could be detected. The urine showed a slight haze in the first glass only. The prostate felt normal though no massage was attempted. Treatment was a problem for the patient lived at a distance and only one visit each week was possible. During the first week he received two further injections of 300,000 units of penicillin in oil-beeswax and an alkaline medicine by mouth, but there was no improvement and on May i 7 th his discharge was worse than ever and his urine was definitely hazy in the first glass. A seven-day course of sulphadiazine, $4 \mathrm{gm}$. daily was then prescribed.

The next day he developed a painful swollen right knee which was followed the day after by a bilateral conjunctivitis. He was kept in bed at home and the sulphadiazine was continued and he attended the clinic again on May 24th. By this time his eyes had cleared but there was a massive effusion present in his right knee and his temperature was $100^{\circ} \mathrm{F}$. Keratodermia, in the form of a greyish-pink raised area with a scalloped edge was also apparent on the glans penis $I-I \frac{1}{2} \mathrm{~cm}$. around the urinary meatus. The discharge was still present, though less; the urine was still hazy and the prostate felt normal. The wife was examined for the second time and found free of all urethral, cervical or vaginal discharge and smears, cultures and her Wassermann, Kahn and gonococcal complement fixation tests were all negative.

Treatment from this point was confined to a potassium citrate mixture and was not subsequently changed, and by June $14^{\text {th }}$, the $43^{\text {rd }}$ day of the disease, the knee lesion had entirely subsided and the keratosis nearly so. He still had a damp urethral discharge and the urine was not entirely normal, though the Wassermann, Kahn and gonococcal complement fixation tests per气 sisted negative.

On August 9th, the patient reported an un $\stackrel{\mathbb{2}}{2}$ pleasant discovery which may have some bearing on the case. Catching an earlier bus from the clinic to his home the week before, he surprisedf his wife with another man, and learnt that this had happened on several occasions before, both $\overline{\bar{n}}$ previous to and after the development of his own symptoms. The wife was again examined and though smears and cultures were still negatives there was a noticeable cervicitis, and the $\overrightarrow{0}$ gonococcal complement fixation test gave a mild one plus result. She was given a single dose of 300,000 units of penicillin in oil-beeswax.

When last seen on September 2oth, the wife both clinically and pathologically was entirelyt negative and the husband, apart from some urethral dampness was also negative.

Though the data is, of necessity, incomplete the definite proved history of marital infidelity seems to offer a better explanation of its origin? than assuming that it arose from an unknown source. It may be that the wife was an asymp-D tomatic carrier of the virus or pleuro-pneumonia like organisms which may be responsible for the disease. If the latter, it is well known that they can abide silently in an apparently normal persam

\section{Summary}

Two cases of Reiter's disease are described. Both were adult males who had suffered from a음 previous urethritis, one gonococcal and one unconfirmed, 12 years before.

In both repeated smears and serological tests $\overrightarrow{7}$ failed to reveal any evidence of gonorrhoea. In neither was there any response to penicillin and with sulphadiazine both developed conjunctivitis, and one a severe synovitis of the knee while receiving the drug. In one the joint lesions were 3 . minimal but both developed a well-marked keratosis of the penis.

In both cases subsequent treatment wasô minimal, one being given only potassium citrate orally and the other three injections of his own? blood.

Both were treated at hospital clinics on an을. out-patient basis, though one had three weeks offi work at his home.

\section{REFERENGES}

I. WILLCOX, R. R., FINDLAY, G. M., and HENDERSON-C BEGG, A. (1947), Brit. Med. Fourn., I, 483 2. HARKNESS, A. H. (1947), Brit. Med. Fourn., I, 612. 3. BAXTER, C. R. (1946), Brit. Med. Fourn., 2, 858 . 4. BAINES, G H. (1947), Brit. Med. Fourn., 2, 605. 